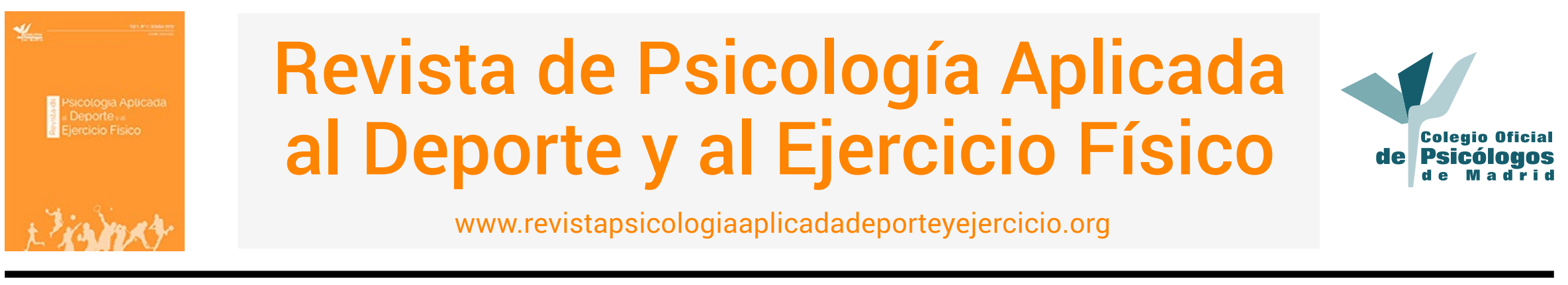

\title{
Entrevista a Joaquín Valdés Fonseca
}

\author{
Ex psicólogo del FC Barcelona, AS Roma y Sporting de Gijón. Actual psicólogo del Real Club Celta de Vigo
}

\section{Por David Peris Delcampo}

Joaquín Valdés pasó de ser un deportista de élite (judo) a convertirse en psicólogo del deporte de uno de los equipos de referencia en el panorama futbolístico mundial. Tras una larga trayectoria profesional, ha desarrollado su función como psicólogo de todas las categorías del Sporting de Gijón (2000-2005), Filial del FC Barcelona (2008-2011), As Roma (2011-2012), Real Club Celta de Vigo (20132014) y el primer equipo del FC Barcelona (2014-2017). Actualmente, en la temporada (2017-2018) ha vuelto al Real Club Celta de Vigo para desempeñar su función como psicólogo miembro del cuerpo técnico del primer equipo

Joaquín, llevas una larga trayectoria como psicólogo del deporte sobre todo en fútbol ¿Qué es lo que te lleva a convertirte en psicólogo del deporte? ¿Cómo empezó todo?

El deporte ha sido y es actualmente el "leitmotiv" que ha marcado toda mi existencia. Ya durante la primera etapa escolar pude practicar distintos deportes: balonmano, fútbol, judo y gimnasia deportiva, lo cual me permitió acabar eligiendo el que más me gustaba en aquel momento, que era el judo. Esto me llevó a ser primero competidor y luego entrenador de este deporte a la vez que llevaba a cabo mis estudios de Psicología. Llega un momento en que hay que decidir cómo uno va a conducir su futuro profesional, y es ahí cuando intento combinar lo que más me gusta, el deporte, con mi futura actividad profesional, la Psicología, a través del Máster de Psicología de la actividad física y el deporte de la UNED, el único Máster que pude realizar en ese momento, allá por el año 1997, el cual supuso un momento clave en mi desarrollo profesional, pues me permitió adquirir los conocimientos y la confianza necesaria para abordar la profesión. Con esto no quiero decir que sea dicho Máster el que haya que cursar para estar bien preparado, pero sí que es necesario buscar en el mercado académico una formación adecuada que esté respaldada por una institución seria.

¿Cómo fue tu incorporación al mundo del fútbol profesional?
El primer contacto que tuve para trabajar con un equipo de fútbol fue en el Real Sporting de Gijón en la temporada 2000-01. En los "Encuentros" de psicólogos del deporte que manteníamos en aquel momento, uno de los temas que se trataba con frecuencia era el de la incorporación del psicólogo a un equipo de fútbol profesional. En mi caso se dio a través del Dr. De La Fuente, Jefe de los Servicios Médicos del Club. Desde bastantes años atrás, la "desgracia" (o la "suerte") de haber sufrido numerosas lesiones e intervenciones quirúrgicas a lo largo del periodo en que fui competidor de Judo, hizo que alcanzara una relación frecuente y hasta hoy de agradecida amistad con dicho Dr. en su clínica privada. Durante el periodo que coincidió con mis estudios de Máster, una de mis múltiples lesiones, me llevó a pasar tardes interminables en su sala de espera que aprovechaba para consultar los libros de dicho Máster. Consciente de que me interesaba que el Dr. De la Fuente supiera de mi especialidad, en una de las visitas dejé "estratégicamente" colocado sobre su mesa uno de los libros con la esperanza de que su curiosidad hiciera el resto. Efectivamente, lo cogió y le echó un vistazo preguntándome qué era lo que estaba estudiando. Cuando lo supo, rápidamente me ofreció presentarme a Jesús García-Barrero (DEP), uno de los pioneros en España como Psicólogo del Deporte, que en aquel momento trabajaba como tal en el Real Sporting de Gijón, con el cual se inició también una buena colaboración.

Unos años más tarde, a consecuencia de sufrir una rotura de LCA (Ligamento Cruzado Anterior), hizo que la asistencia diaria para hacer la rehabilitación en su clínica, me 
permitiera presentarle un proyecto de trabajo orientado a la intervención psicológica en la rehabilitación de lesiones deportivas, siendo contratado para trabajar como psicólogo con los pacientes que tenían que operarse de LCA. Al cabo de un tiempo, cuando el Dr. De La Fuente lo consideró oportuno, propuso al Club la necesidad de incorporar de nuevo la figura de un psicólogo, y ahí se inició mi incorporación al fútbol profesional.

Desde el año 2000 hasta el 2005, fue el periodo en que se desarrolló esta etapa. Como es lógico, eran muchas las dudas que me asaltaban a la hora de desarrollar por primera vez un proyecto de esta envergadura (cómo negociar mi contrato, cuánto tiempo de dedicación, cómo distribuirlo,...). Básico para mí fue poder compartir inquietudes con otros compañeros de profesión que fueron de gran ayuda en aquel momento. Disponer de un buen círculo de relaciones profesionales es algo que debemos cuidar e impulsar siempre. José $\mathrm{M}^{a}$ Buceta (director del Máster en Psicología del deporte de la UNED), fue un ejemplo de ello. Su apoyo en aquel momento fue de gran ayuda. Me puso en contacto con Pedro Almeida, psicólogo del Benfica, el cual amablemente me acogió durante una semana en Lisboa, poniendo a mi disposición tanto sus conocimientos como su forma de trabajar. Este tipo de apoyo resulta "impagable".

He tenido especial interés en desarrollar toda esta explicación anterior, para resaltar la importancia que puede tener en un momento dado, ante una situación aparentemente inocua, el hecho de "dejar caer" a personas "clave", quién eres, qué haces, dónde,... Muchos me han dicho en más de una ocasión "qué suerte tienes, pudiendo trabajar en...". La cuestión es: ¿ha sido suerte? O te lo has buscado. Realmente pienso, que no estoy seguro de qué día fue el que di los primeros pasos para incorporarme a un equipo de fútbol profesional. Puede haber sido el día que entré en las instalaciones de la Escuela de fútbol de Mareo, o quizás el día que dejé el libro del Máster encima de la mesa del Dr. De La Fuente, o el día que decidí estudiar el Máster, o el día... De lo que estoy seguro es que todos los pasos que damos en nuestras vidas tienen una repercusión en el futuro, por lo que tener más o menos claro lo que persigues te va a ayudar a encontrar el camino. La "suerte" de alcanzar lo que tú quieres depende en gran medida de ti.

A partir de tu incorporación en el Real Sporting de Gijón, empiezas a desarrollar tu trabajo como psicólogo del deporte en un club de fútbol. ¿Cómo siguió tu trayectoria profesional?

En la temporada 2000-01, tengo el primer contacto para trabajar con el fútbol profesional a nivel institución. Antes ya lo había hecho pero sólo con jugadores a nivel individual. Fue en el Real Sporting de Gijón, equipo de mi ciudad, donde permanecí hasta la temporada 2004-05, momento en el que se produce un "proceso concursal" en el Club, que hace que nos quedemos fuera 14 personas. Durante el tiempo que permanezco aquí, debido a la situación económica del Club, el horario de dedicación a mi trabajo, varía a lo largo de las distintas temporadas, siendo en el mejor de los casos de media jornada, lo cual siempre me obligó a combinarlo con otros trabajos al margen del fútbol. En el caso del Sporting, mi figura como psicólogo formaba parte de la institución, no exclusivamente del primer equipo, aunque éste siempre fuera la prioridad. El $50 \%$ del tiempo era dedicado al primer equipo y el otro $50 \%$ a las categorías inferiores.

En la temporada 2008-09, fui convocado por el Fútbol Club Barcelona, en la figura del que iba a ser el entrenador del equipo filial, Luis Enrique Martínez, para hacerme una entrevista de trabajo. Una vez superada dicha entrevista, ésta constituyó el inicio de una forma de trabajar diferente, pues a partir de ese momento pasaba a formar parte su cuerpo técnico, acompañándolo tres temporadas en dicho filial (2008-09 a 2010-11), una en la AS Roma (2011-12), otra en el Real Club Celta de Vigo (2013-14) y tres más en el Fútbol Club Barcelona (2014-15 a 2016-17). Como es lógico, las características del trabajo formando parte de un cuerpo técnico, o formando parte integrante de la estructura de un club contienen diferencias notables.

En la actualidad (2017-18) vuelvo al Real Club Celta de Vigo, en esta ocasión a las órdenes de otro entrenador, Juan Carlos Unzué, formando parte de su cuerpo técnico.

Trabajaste en la base del Sporting de Gijón, donde además compaginabas tu labor con el filial y el primer equipo. ¿Qué es lo que hiciste como psicólogo del fútbol base?

Como psicólogo del fútbol base intenté dirigirme a los tres grandes ámbitos que eran los entrenadores, padres y jugadores, lo que era el triángulo deportivo. Organicé un programa de valores, refuerzos, consecuencias... donde trabajamos la filosofía del club por medio de Sportings, que era un sistema de puntos (economía de fichas) en el que, utilizando el acrónimo del nombre del club, se trabajaban diversos valores que eran: (S) Salud, Seguridad e higiene, $(P)$ Puntualidad, (O) Orden, (R) Respeto, (T) Tenacidad y esfuerzo, (I) Imagen, (N) Nobleza y dignidad y (G) Grupo.

Con los entrenadores, una parte importante del trabajo fue su formación en cómo llevar a cabo el desarrollo e implantación de este programa (explicaciones en grupo, reuniones individuales, observación de entrenamientos,...), todo 
lo que eran las directrices de funcionamiento del Sporting de Gijón y los "Sportings".

Con los padres, el objetivo principal era el asesoramiento en el deporte de sus hijos. Para ello organizamos charlas de distinta índole. A principio, mediados y final de temporada junto con el secretario técnico y el gerente del club, trabajando sobre lo que se esperaba de ellos, y lo que ellos podían esperar de un Club como el Sporting de Gijón. Diferentes charlas también implicando a nutricionistas, médicos, etc. en las que se fomentase una buena educación en ese sentido.

Con los niños la intervención principal se llevó a cabo de forma indirecta, a través de los entrenadores, y de forma individual en casos concretos en los que se había detectado la necesidad. Este tipo de intervención era muy complicada de generalizar debido al gran número de niños que integraban el fútbol base y la limitación de tiempo de dedicación por mi parte.

¿En el primer equipo trabajabas con jugadores de manera individual, por grupos, con el entrenador...?

Tanto con el filial como con en el primer equipo, mantuve una estructura parecida de trabajo, manteniendo un contacto permanente tanto con el entrenador como con el resto de los miembros del cuerpo técnico. Desde aquellos días hasta la actualidad, siempre he mantenido una idea muy parecida en mi forma de trabajar, que principalmente ha variado, y esto es a mi modo de ver un aspecto muy importante, en función del entrenador con el que esté trabajando. Una de las virtudes más importantes que considero debe tener un psicólogo del deporte es su "capacidad de adaptación".

A principio de temporada hacíamos reuniones con jugadores del primer equipo para trabajar temas específicos como comunicación, temas de relajación para reducción de la carga de pretemporada, etc... trabajando de manera individual o en grupos reducidos a demanda. También hacía talleres semanales de manera voluntaria trabajando con ellos sobre todo habilidades psicológicas como la respiración, control de la activación, entrenamiento en imaginación, exposición de temas en los que a través de preguntas llevarles a la auto-reflexión (tipo coaching)... muchas de aquellas cosas (de hace 18 años) las sigo utilizando actualmente.

Uno de los primeros objetivos que me planteé en aquel momento fue el de integrar la figura del psicólogo en el día a día del equipo, para que se normalizase y no resultara un elemento extraño en la convivencia del día a día del equipo. Desde entonces hasta la actualidad es algo que se ha conseguido, pues cada vez es más frecuente nuestra figura en el mundo del deporte en general y en el del fútbol en particular.

Es cierto que en el Sporting estuvo Jesús García Barrero y Rosana Llames anteriormente; pero entre ellos y yo pasaron unos años en los que ya no seguían las mismas personas, los mismos jugadores... y había que seguir rompiendo barreras.

A nivel individual hubo jugadores que tanto las temporadas que estuve trabajando en el Sporting como después cuando ya no estaba allí, continuaban trabajando de forma rutinaria todas las semanas viéndonos en mi consulta privada. Recuerdo que en una ocasión, uno de los entrenadores con los que trabajé, me presentó al equipo diciendo "Este es Joaquín, el psicólogo. Os puede ayudar cuando tengáis algún problema". Cuando escuché cómo me presentó, fui consciente de que yo no le había explicado bien cuál era mi función, pues yo no quería que asociasen mi figura exclusivamente a "si problemas - entonces psicólogo". El primer paso fue hacer que los entrenadores supieran que nuestra ayuda no era sólo para cuando tuvieran problemas, sino sobretodo para conseguir mantener el rendimiento al mejor nivel. Siempre utilicé un ejemplo que tanto jugadores como entrenadores entendían muy bien para trazar un paralelismo, el de la función del Preparador Físico: Ps - ¿Cómo te encuentras físicamente? /Jugador - Bien / Ps - Entonces, si ya estás bien ¿porqué sigues haciendo trabajo físico? / Jugador - Para mantenerme físicamente lo mejor posible / Ps - Pues el trabajo psicológico es igual, aunque estés a un buen nivel en un momento dado, entrenar psicológicamente te ayudará a rendir durante más tiempo. También de la misma forma, cuando por el motivo que sea tienes problemas físicos, necesitas trabajo diferente al de tus compañeros con el preparador físico que te ayudará a recuperarte. Lo mismo ocurre si estos problemas son psicológicos, el psicólogo te ayudará también a recuperarte.

Conseguir que se asociase también nuestro trabajo a la optimización del rendimiento fue uno de los objetivos que perseguía y creo que se consiguió en gran medida.

Después, tu experiencia como psicólogo del filial del FC Barcelona, tanto en División B como en la División de Plata del fútbol español. Llegas a un "grande", a su filial de la mano del primer técnico y permaneciendo hasta tres temporadas seguidas. ¿Qué aspectos puedes destacar de tu trabajo durante esos tres años?

El trabajo en el filial del FC Barcelona supuso un cambio importante en mi desarrollo profesional. La dimensión del nuevo equipo, otra ciudad, toma de decisiones a nivel familiar y, sobre todo un nuevo entrenador, Luis Enrique Martínez, que marca un antes y un después en mi trayectoria a partir de ese momento. Un entrenador adelantado a su tiempo, del cual no resulta extraño que haya conseguido triunfado tanto de jugador como de entrenador. Tuvo muy claro desde su inicio profesional como entrenador la incorporación de un 
psicólogo a su cuerpo técnico. Siempre admitió públicamente la importancia que para él tiene esta figura ya desde su época de jugador. Para esta incorporación se llevó a cabo una selección con diferentes psicólogos siendo yo al final el profesional elegido. Considero que ha sido él, si no el que más, de los que más impulso le ha dado a nuestra profesión en el mundo del fútbol.

Aquí llevé a cabo un trabajo mucho más cercano al entrenador, de mucha más continuidad, donde tenía mucha influencia la forma de trabajar del técnico. Hasta aquel momento (2008) y también bastante después, el despacho de los entrenadores solía ser el mismo vestuario en el que se cambiaban. A partir de ese momento el trabajo con el entrenador y todo el cuerpo técnico se lleva a cabo en un despacho amplio donde estamos todos juntos. Comienza mucho antes de salir al césped y continúa mucho después de acabar los jugadores. Lo que quiere el entrenador es que tanto el $2^{\circ}$ entrenador, como el psicólogo, como el preparador físico estén trabajando conjuntamente en continua comunicación, de manera que cada uno aporta lo que le corresponde a su parcela cuando se trata un tema, haciendo una puesta en común. Este estilo de trabajo se mantuvo siempre, y actualmente con Juan Carlos Unzúe trabajamos de la misma manera, cada uno aportando lo que le corresponde.

A partir de este momento estoy en los entrenamientos, en los partidos, en los desplazamientos..., conviviendo siempre como uno más del cuerpo técnico. Ello me permite poder observar en directo los estados de ánimo de los jugadores, las habilidades psicológicas que manejan, la capacidad de adaptación, el clima de trabajo, la cohesión, los estilos de comunicación,... información necesaria para poder ayudar tanto a los jugadores como al entrenador, que de otra forma sería muy difícil de recabar.

En el Barça B fueron tres las temporadas que permanecí trabajando. La dedicación fue de tres días por semana al principio, pero poco a poco la demanda fue aumentando hasta llegar a ser prácticamente completa. Esto también me ocurrió en el Sporting de Gijón, donde la demanda también fue de menos a más.

Inicias una nueva etapa, esta vez en el fútbol profesional y en un país extranjero, con un idioma diferente y en una liga quizás también diferente. En tu experiencia en A.S. Roma, ¿cómo te adaptas y qué haces en este nuevo equipo?

Otro aspecto muy formativo para mí, se dio en el intervalo que va desde que acaba nuestra relación con el Barça B hasta llegar a firmar con la AS Roma. Pude experimentar la incertidumbre que se vive en el mundo del fútbol cuando se está pendiente de los posibles equipos a los que puedes ir, si se llega a un acuerdo o no, si llegarás a trabajar la siguiente temporada o no...

Mi dedicación en la AS Roma, como en el Barça B, también fue poco a poco en aumento. Lo primero aprender italiano para poder comunicarme, aunque suponía una dificultad para poder trabajar al mismo nivel que en castellano. En cualquier caso, en el equipo había varios jugadores de habla castellana (argentinos y españoles), lo cual facilitaba la labor. También fui demandado por el club para trabajar con jugadores de habla hispana que había en el equipo filial.

En la AS Roma Luis Enrique formó un cuerpo técnico que ha continuado en las temporadas siguientes hasta la actualidad. Está compuesto por el segundo entrenador Robert Moreno y el preparador físico Rafel Pol (más tarde, ya en el RC Celta de Vigo también se incorpora Juan Carlos Unzué). Juntos llevamos trabajando más de seis años. Esto conlleva que no sólo haya trabajado con el primer entrenador sino también con el resto del cuerpo técnico ante situaciones potencialmente estresantes como vivir en un país diferente, temas familiares, temas deportivos, de comunicación con los jugadores...

Uno de los aspectos más importantes de mi trabajo a lo largo de estos años, ha sido el cuidado del cuerpo técnico, nuestras relaciones internas. En un trabajo como el de un cuerpo técnico, son muchas las horas de convivencia que nos vemos obligados a compartir (viajes, pretemporadas, concentraciones,...) lo cual puede afectar inevitablemente a nuestro rendimiento. A lo largo de estos años, no sólo hemos conseguido un equipo de trabajo eficiente, sino además una buena relación de amistad que hace que podamos disfrutar aún más de nuestro trabajo diario, manteniendo unos lazos muy potentes en los momentos más duros. Con esto no quiero decir que es necesario entablar amistad con tus compañeros de trabajo, pero no cabe duda que cuando se disfruta de ella el rendimiento es mucho más alto. La confianza que han tenido en mi trabajo, nos ha permitido mantener un buen equilibrio interno.

Vuelves al fútbol español, a La Liga y a un primer equipo, al Real Club Celta. ¿Qué diferencias encuentras en tus equipos anteriores?

Cuando finaliza la temporada en la AS Roma, Luis Enrique decide descansar, volviendo a reunirnos un año después para fichar por RC Celta de Vigo.

En el Celta continúo trabajando de forma muy similar a los equipos anteriores. Al trabajo diario con el cuerpo técnico, se suma la recuperación del trabajo con los jugadores a nivel colectivo y con pequeños grupos que en la AS Roma no había podido llevar a cabo. 
En pretemporada se incluyen en la planificación varias sesiones de trabajo psicológico con todo el equipo, con actividades tanto indoor como outdoor en las que a través de la consecución de pequeños retos nos permiten llegar a conclusiones acerca de la importancia del trabajo en equipo, la comunicación, el compromiso, etc. También aprovechando este periodo de concentración antes del inicio de temporada, se llevó a cabo una entrevista individual de toma de contacto con todos y cada uno de los jugadores, con el objetivo tanto de recibir información como de informarles acerca del trabajo psicológico.

Durante la temporada, dos veces por semana trabajábamos la recuperación del partido anterior o bien la preparación del partido siguiente, previa formación en habilidades psicológicas (control de la activación, respiración, entrenamiento en imaginación...), con un grupo reducido de jugadores.

También se programaron jornadas de convivencia de tipo lúdico, potenciadoras de la cohesión, que a su vez permitían observar muchos aspectos de comportamiento, relacionales, etc., dando mucha información.

Llegas al cuerpo técnico del primer equipo de un club como el FC Barcelona y el primer entrenador, en su primera conferencia, te presenta como el psicólogo y dice algo así como "tranquilos, que el psicólogo es para mí". ¿Cómo valoras tu incorporación a un primer equipo como el FC Barcelona y cómo consideras tu experiencia?

Sí, ese fue uno de los titulares del momento y toda una declaración transparente de la importancia que el entrenador le daba a la figura del psicólogo. Con cierto tono sarcástico, pero con un mensaje claro.

Cuando hablas del FC Barcelona todo es noticia, lo que es importante y lo que no lo es. En este sentido muchas de las declaraciones de Luis Enrique ante los medios eran en la dirección de proteger a las personas que estábamos alrededor. Cuando estás en un equipo de esta envergadura, no es que sea más difícil trabajar pero sí que hay que estar muy pendiente de cosas que en otros equipos no es tan necesario. Las cámaras siempre están preparadas para inmortalizar cualquier comentario, movimiento de labios, gesto, acercamiento a un jugador, ... para luego ser interpretado como más convenga para aumentar el número de lectores. ¿Cuál es la clave para trabajar en sitio así? De nuevo "adaptación". No hay que luchar contra ello, hay que trabajar con ello.

En un equipo como el FC Barcelona se multiplica el número de personas a gestionar, el número de cosas a tener en cuenta cada día. Se multiplica también el intento de filtrar al entrenador lo exclusivamente necesario para evitar "quemarlo" en pequeñas decisiones, por lo que trabajamos con todo el cuerpo técnico para determinar diferentes parcelas de información y que al primer entrenador le llegue lo estrictamente necesario para que llegar a la competición lo más "fresco" posible.

Es todo multiplicativo. Multiplicas el impacto mediático, multiplicas las horas de convivencia, multiplicas el número de partidos, multiplicas todo porque es un equipo que está jugando cada tres días.

¿Cómo fue tu trabajo como psicólogo del primer equipo del FC Barcelona durante esas tres exitosas temporadas deportivas?

En esencia, el trabajo no fue muy diferente al que he realizado en cualquier otro equipo. La cuestión fue adaptarlo al contexto.

En pretemporada, al igual que en otros equipos anteriormente, también se realizaron actividades conjuntas programadas en la planificación como un entrenamiento más. A través de la convivencia, "de estar ahí", la captación de información importante que te da la observación en el día a día

Durante la temporada el trabajo con jugadores se produjo a demanda individual. La mayor parte del trabajo en estos periodos fue dirigido a la gestión, apoyo y asesoramiento tanto al entrenador como al resto del cuerpo técnico.

Por ejemplo, en el tema de prensa, al jugarse tres partidos a la semana, había una rueda de prensa previa y otra post-partido. Una recomendación que siempre he hecho tanto a jugadores como a entrenadores ha sido evitar leerla. Es muy agradable cuando hablan bien de ti, pero aún no conozco a nadie al que no le afecte cuando es al contrario, por mucho que se empeñen en manifestar públicamente que no es así. De nada sirve estar intoxicándose con titulares cada día, que pueden afectar al estado emocional y por tanto a nuestro rendimiento. Se trata de no transmitir al entrenador todo lo que sale en prensa, sino de hacerlo sólo con lo estrictamente necesario. Lo que realmente importa y que puede tener efecto sobre el rendimiento, o bien información que no importa tanto, pero conviene que esté al tanto de ella. En un club del nivel del FC Barcelona, la parte del impacto mediático consume una parte importante de energía.

Una función muy importante de la que nos encargábamos todo el cuerpo técnico, era la de estar muy atentos a resolver problemas o tomar decisiones que estuvieran en nuestras manos, para evitar que tuvieran que llegar al entrenador, y así reducirle carga ya de por sí alta que debía resolver, con el objetivo de que pudiera llegar al día del partido lo más "fresco" posible (temas de viajes, comedor, seguimiento de normas, ...) 
Has comentado que realizas un trabajo individual y grupal con jugadores sobre diferentes temas, entre los que está el entrenamiento en imaginación. ¿Podrías explicar cómo realizas ese trabajo?

Una de las Habilidades psicológicas que he trabajado desde siempre en todos los equipos que he estado, es la del entrenamiento en imaginación. En ocasiones de forma individual y en otras con pequeños grupos. Siempre de forma voluntaria, pues considero que si no hay una buena predisposición por parte de quien lo recibe, es muy difícil beneficiarse de él.

Ahora mismo, en el equipo que estoy trabajando, hay un grupo de jugadores que lo entrena todas las semanas, generalmente el día antes de partido. En función del tiempo del que disponemos, se hace al final del entrenamiento o bien en el hotel de concentración. Dura unos 10 minutos aproximadamente.

Para poder llegar a esta sesión grupal, antes ha habido varias sesiones de formación previas a nivel individual, en las que han tenido que aprender otras habilidades psicológicas como la respiración, la relajación, cómo elaborar la conducta objetivo, imaginar en primera persona,... también muy importante, es hacerles conocedores de porqué ese tipo de entrenamiento es beneficioso para ellos, explicándoles el funcionamiento de la herramienta y los objetivos de control del nivel de activación, autoconfianza o atención que se persiguen. Como se puede observar, para poder hacer una sesión de entrenamiento en imaginación, se requiere un amplio trabajo previo.

Una vez realizado todo ese trabajo, los pasos que llevamos a cabo en una sesión de entrenamiento en imaginación son:

1. Elegir la conducta-objetivo que se desea ensayar (pase de balón, presión, algún momento concreto en el vestuario antes de empezar, antes del entrenamiento...)

2. Elegir el lugar donde se produce (campo, zona concreta del campo, vestuario, ...), lo que tengo a mi derecha e izquierda, el clima, la temperatura, la hora del día, el olor que hay en el ambiente. Debe estar todo previsto, sin dejar cosas al azar.

3. Elaborar el "PLAN ATENCIONAL" : a) ¿Qué ocurre justo antes de la conducta-objetivo?. (Posición del compañero, posición del contrario,...) b) Ensayar la conducta-objetivo (pase de balón, regate, control, disputa...) c) Desenlace positivo.

4. Realizando respiración o relajación.

5. Hacer 4 series de entre 30 y 40 segundos repitiendo el plan atencional. Intercalar ejercicio de respiración de 30 segundos aproximadamente entre serie y serie.
6. Finalizar con un minuto, situándose mentalmente en la competición e imaginándose a sí mismo disfrutando, haciendo buenas jugadas, sintiendo que se domina la situación.

En las primeras sesiones les suelo entregar un registro con las instrucciones anteriores y un anexo para que describan los planes atencionales y así poder analizarlos conjuntamente para corregirlos si fuera necesario.

\section{Plan atencional 1: (ejemplo)}

a) (Recibo el balón de un compañero en zona de finalización)

b) (Me giro hacia el contrario y me voy de él)

c) (Tiro a puerta)

Lugar: (Balaídos)

Clima: (Sol)

Temperatura: (Algo de calor)

Olor: (a césped)

\section{Plan atencional 2:}

a) (mi compañero $X$ recibe el balón)

b) (me coloco detrás del contrario y en diagonal con mi compañero X. Recibo el balón sólo y juego con mi compañero Z)

c) (Mi compañero Z recibe el balón en buenas condiciones) Lugar: (San Mamés. Atacando hacia la portería que está a la derecha de los banquillos)

Clima: Lloviendo

Temperatura: Frío

Olor: Césped

El trabajo durante las series se distribuye así:

\section{Serie 1}

1) 40 seg repitiendo una y otra vez plan atencional 1

2) 30 seg centrado en la respiración

\section{Serie 2}

1) 40 seg repitiendo una y otra vez plan atencional 1

2) 30 seg centrado en la respiración

Serie 3

1) 40 seg repitiendo una y otra vez plan atencional 2

2) 30 seg centrado en la respiración

\section{Serie 4}

1) 40 seg repitiendo una y otra vez plan atencional 2

2) 30 seg centrado en la respiración

Cuando ya dominan la técnica, ellos por su cuenta deciden qué conductas objetivo desean trabajar y las van cambiando en función de lo que consideran necesario para el siguiente partido. Lo que yo hago es dirigir la sesión. Lo hacemos primero reduciendo el nivel de activación a través de la respiración y un breve recorrido del cuerpo para 
relajarlo. A continuación nos desplazamos mentalmente al lugar donde quieren que se produzca la conducta objetivo pidiéndoles que busquen qué es lo que tienen a su derecha, a su izquierda, la temperatura, hora del día, ambiente, el campo donde están... y a continuación cuando estén preparados les indico: "cuando diga ya iniciaremos la primera serie de la conducta objetivo"... "YA", ....... Cuando han pasado 40 segundos les indico que ha terminado la serie diciendo de nuevo: "YA"..."cambio de atención a la respiración...recuperándonos de la primera serie" durante 30 segundos se centran en la respiración, una atención neutra que les permite recuperarse hasta que les avise del comienzo de la segunda serie, continuando así durante cuatro series. Después de la cuarta serie, se imaginan durante un minuto de forma libre, haciendo buenas jugadas, buenos movimientos, buenos pases,... hasta que les indique el final de la sesión.

Es muy importante en esta técnica no dejar nada al azar, buscar el control sobre todas las acciones que vamos a imaginar para evitar que se pueda generar cualquier pensamiento negativo.

Ahora mismo, hay muchas personas, muchos de ellos psicólogos, que les gustaría incorporarse como profesionales "mentales" en el mundo del fútbol. ¿Qué les puedes decir a estas personas?

En primer lugar, y lo más importante, que se formen adecuadamente. Buscar en el mercado académico una formación adecuada que esté respaldada por una institución seria. A lo largo de estos años, han sido muchas las personas que se me han acercado para pedir consejo en su inicio como psicólogos deportivos y otras, ya trabajando con deportistas, para intercambiar opiniones. Para mi asombro en la gran mayoría de los casos, sin una formación sólida que respalde sus conocimientos, o simplemente con algún "pseudo-curso" realizado de esos que proliferan hoy en día.

Cuando nos enfrentamos a la situación laboral ante un equipo de fútbol, o cualquier otro deporte, tenemos que tener en cuenta la responsabilidad que asumimos, no sólo con la entidad, sino también con nosotros mismos y con nuestra profesión. Con la Entidad deportiva desde el punto de vista de lo que pueden esperar de nosotros; con nosotros mismos desde el punto de vista de tener la confianza de estar preparados para nuestro desempeño; y con nuestra profesión desde la perspectiva de dignificarla y seguir abriendo puertas a los que vienen detrás. Huyo de seguir teniendo que justificar nuestro trabajo como me ocurría hace años, pero nuestra profesión continúa siendo lo sufi- cientemente joven como para estar muy pendiente de dignificarla. Para ello no veo otra vía que la de la buena praxis respaldada por una formación sólida.

El Colegio de Psicólogos, a través de la División de Psicología del Deporte, ha puesto en marcha la acreditación de Experto en Psicología del Deporte. ¿Qué te parecen estas iniciativas y qué cosas podríamos hacer al respecto para favorecer una buena práctica profesional desde la psicología del deporte?

Dada la situación actual, en la que existe un elevado intrusismo en nuestra profesión, me parece una muy buena iniciativa que un organismo serio y respetado, como es un Colegio Profesional, describa y acredite de forma oficial las características que debe recoger una persona para estar capacitada como Psicólogo del Deporte. El siguiente paso creo que debería ser, difundir la existencia de dicha acreditación en todos los contextos susceptibles de realizar la contratación de un psicólogo deportivo. Cuanta mayor difusión, mayor probabilidad de conseguir que nuestra especialidad avance en la dirección correcta.

Muchas gracias, Joaquín por tu tiempo y tu entrevista. Te deseamos lo mejor en tus futuros proyectos. 